\title{
Cancer Pain: From Molecules to Suffering
}

\author{
Judith A. Paice, Rae F. Bell, Eija A. Kalso, Olaitan A. Soyannwo. IASP Press; \\ International Association for the Study of Pain, 2010, 354 pages. \\ ISBN 978-0-931092-81-7
}

\author{
Christopher Green, MBBCh
}

Published online: 7 December 2010

(C) Canadian Anesthesiologists' Society 2010

Cancer Pain is an up-to-date account of the current scientific and clinical findings relating to cancer pain. The book was written in response to an international symposium sponsored by the International Association for the Study of Pain that featured speakers regarded as experts in their field. Each of the clearly written chapters is based on a speaker's presentation and is well referenced, analogous to a review article on the relevant topic. Also, despite some overlap, the chapters can be read together or independently. Essentially, the book offers readers a wide-ranging review of the issues surrounding cancer pain management and the direction of future research.

The book is divided into six sections. The first of these deals with the basic mechanisms of cancer pain with regard to chemotherapy-induced neuropathic (CIN) pain and bone pain. It addresses the incidence of CIN pain with reference to modern chemotherapeutic agents and evidence that protein kinases, inflammation, direct cytotoxicity, and deficiencies in growth factor play a role as possible co-existing pathogenic mechanisms. There is also a brief description of the role played by transient receptor potential vanilloid and the $\alpha 2 \delta 1$ subunit of voltage-dependent $\mathrm{Ca}$ channels in the generation of sensitization. Clinical findings of CIN pain and its likely course of development and prognosis are described together with typical findings on sensory motor testing. The currently available pharmacological therapies are discussed, while acknowledging that most lack efficacy. The chapter on bone pain demonstrates how animal and human modelling are providing insight into the mechanisms of bone pain, and it examines clinical trials of novel therapies targeted towards these

C. Green, MBBCh $(\bowtie)$

The Ottawa Hospital, Ottawa, ON, Canada

e-mail: kitgreen@doctors.net.uk mechanisms, including bisphosphonates, anti-receptor activator for nuclear factor and Kappa B ligand and antinerve growth factor therapy, and pregabalin. These drugs are further discussed in a later chapter where novel agents are considered and their preclinical and early clinical trials are outlined. There is also a chapter describing the technical, biological, and practical aspects of radiotherapyinduced analgesia, with discussion of its effectiveness in differing types of cancer.

The second section deals with the role of inflammation in cancer pain, with particular reference to cytokines (e.g., tumour necrosis factor $\alpha$, interleukin- $1 \beta$, interleukin- 6 , and others) and their role in hypersensitivity and hyperalgesia. It also introduces the concept of the role of inflammation in general in the generation of cachexia and symptom clusters (e.g., pain, depression, and fatigue) associated with cancer.

The third section deals with the use of opiates in cancer pain and begins with the issue of opioid-induced hyperalgesia and its potential mechanisms. It also highlights early work showing the potential implications of bolus vs continuous delivery in enhancing cancer-induced pain in animal models and in altering tumour growth, bone loss, and fracture. The role of co-analgesics is also discussed. The current theories behind opioid tolerance are then explored, including explanations of the mechanisms of receptor desensitization, internalization and recycling, G-protein signalling, and dual receptor targeting. Methods of managing this phenomenon clinically are discussed, including opioid switching, with mention of the available evidence for its efficacy and practical problems associated with its implementation. Drugs acting against tolerance are also dealt with, in particular, N-methyl-D-aspartate receptor antagonists, steroids, opioid antagonists, adrenoceptor antagonists, antagonists of anti-opioid peptides, and inhibitors of glial activation. 
The fourth section concentrates on the methodological issues associated with clinical trial design for both pharmacological and non-pharmacological interventions for cancer pain.

The penultimate part of the book is concerned with the psychology surrounding cancer pain. The relationship between anxiety and pain is dealt with as well as how pain alters attention to cause interruption, interference, and a threat to the sense of identity. The authors address the concept of attention as a "limited channel capacity". They describe the way attention can be manipulated in practice through Cognitive Behavioural Programs or use of hypnosis and provide evidence for the efficacy of these interventions. Partner-assisted coping skills are also addressed, as is the role of empathy (both its benefits and problems) in managing patients.
The remaining chapters emphasize the continued need to improve cancer pain management from a global standpoint through further education of future clinicians and implementation of existing guidelines as supported by current evidence.

In summary, this well written, predominantly scientific review by experts conveys up-to-date information on the subject of cancer pain. The book is not written specifically for anesthesiology or pain medicine practitioners, as there are no sections devoted to the interventional management of chronic cancer pain, which would generate more interest from this peer group. Nonetheless, the book provides interesting background reading and is to be recommended, particularly for those with cancer pain patients in their care. 